\title{
Improved disease-free survival with adjuvant radiotherapy in early-stage endometrial cancer: 10-year outcome analysis
}

\author{
Suheyla Aytac Arslan, MD!, Gulhan Guler Avcı, MD², Ebru Atasever Akkas, MD³, Prof. Yıldız Guney, MD4 \\ 'Department of Radiation Oncology, Faculty of Medicine, Yıldırım Beyazit University, Ankara, Turkey, ${ }^{2}$ Department of Radiation Oncology. \\ Faculty of Medicine, Gaziosmanpaşa University, Tokat, Turkey, ${ }^{3}$ Department of Radiation Oncology, Dr. Abdurrahman Yurtaslan Ankara \\ Oncology Training and Research Hospital, Ankara, Turkey, ${ }^{4}$ Department of Radiation Oncology, Ankara Memorial Hospital, Ankara, Turkey
}

\begin{abstract}
Purpose: The purpose of this study was to evaluate long-term treatment outcomes and prognostic factors affecting survival of patients with early-stage endometrial carcinoma.

Material and methods: Data of 311 patients with FIGO stage I-II endometrial cancer, curatively treated at two different tertiary centers between June 2001 and December 2016 were retrospectively reviewed. The patients had primary surgery, $74(24 \%)$ received no further treatment, 4 (1\%) obtained chemotherapy only, 234 (75\%) received radiotherapy, and $24(7 \%)$ received both.

Results: Median follow-up time was 102 (range, 3-205) months. During this period, 68 (21.9\%) patients died. 5-year and 10 -year disease-free survival (DFS) were $76 \%$ and $74.3 \%$, respectively. In multivariate analysis, lower uterine segment invasion positivity and no adjuvant radiotherapy were determined as independent unfavorable prognostic factors for DFS. The 5-year and 10-year disease-specific survival (DSS) were $86.8 \%$ and 82.2\%, respectively. For DSS, high-grade, lymphovascular space invasion positivity, stage II, $\geq 65$ age, and no adjuvant radiotherapy were found to be independent unfavorable prognostic markers.

Conclusions: The findings of our cohort have confirmed the importance of adjuvant radiotherapy on long-term early-stage endometrial carcinoma outcome.

Key words: early-stage endometrium cancer, adjuvant therapy, radiotherapy, survival.

\section{Purpose}

Endometrial cancer is the second most frequent gynecological malignancy worldwide, and its prevalence is more common among high-income countries [1]. Most of these cases are diagnosed at an early-stage due to post-menopausal vaginal bleeding as an early warning sign [2]. Total abdominal hysterectomy and bilateral salpingo-oophorectomy with or without lymphadenectomy is the mainstay of management. The type of surgery, especially in terms of lymphadenectomy, varies depending on the tumor size, grade, and depth of myometrial invasion of the tumor [2,3]. Post-operative radiotherapy (RT), either external beam RT (EBRT) and/or vaginal brachytherapy (VBT), is recommended for patients with early-stage disease based on the presence of adverse risk features $[4,5,6]$. The results of multiple randomized trials demonstrated a locoregional control benefit with adjuvant RT over observation alone, but its effect on survival is controversial $[7,8]$.
In this study, we aimed to investigate a long-term survival outcomes and prognostic factors affecting survival in patients with early-stage endometrial cancer.

\section{Material and methods}

Patients diagnosed with non-metastatic uterine adenocarcinoma who underwent total hysterectomy and bilateral salpingo-oophorectomy between 2006 and 2016 at two institutions were analyzed. The patients were restaged according to the 2009 International Federation of Gynecology and Obstetrics (FIGO) staging system, and data of 311 patients with stage I-II endometrial cancer who received \pm adjuvant treatment were included and retrospectively reviewed. The patients' files, electronic information system, RT records, and telephone interviews were used to collect the data. The collected and analyzed details included age at diagnosis, date of surgery, type of surgery, inclusion of lymph node dissection, histology,

Address for correspondence: Gulhan Guler Avc1, Department of Radiation Oncology, Faculty of Medicine, Received: 09.03 .2020 Gaziosmanpaşa University, 60100, Tokat, Turkey, phone: +90-546-462-22-82, fax: +90-356-212-00-46, 
grade, depth of myometrial invasion, presence of lymphovascular space invasion (LVSI), type of adjuvant chemotherapy, type of adjuvant radiation, dose of radiation, dates of radiation, date of recurrence, type of recurrence (if applicable), date of last follow-up, and date of death.

All the patients underwent primary surgical treatment, as total abdominal hysterectomy and bilateral salpingo-oophorectomy \pm bilateral pelvic paraaortic lymph node dissection. Post-operative RT either EBRT and/or VBT were suggested to patients with adverse risk factors. Patients with high-grade histologies (non-endometrioid and endometrioid grade 3 ) and stages beyond Ib usually received both the treatments, patients who presented one major risk factor usually obtained either VBT or EBRT, and patients with minor risk factors received no further therapy. Starting from late 2008, the patients were treated with 3D pelvic EBRT, but prior to 2008, a 2D box technique was performed. The linear accelerators with $6 \mathrm{MV}$ or 15-18 MV energies were used at standard fractionation (180-200 cGy) to a total dose of 4,500-5,040 cGy. The anterior/posterior pelvic field borders were L4-L5 vertebra intersection superiorly, below the obturator foramen inferiorly, and $1.5-2 \mathrm{~cm}$ lateral to the widest portion of bony pelvis laterally. The lateral field borders were posterior of S3 and anterior part of pubic symphysis.

Vaginal brachytherapy was applied either as boost therapy following EBRT or as a sole treatment modality. Total VBT dose was 10-25 Gy/2-5 fractions prescribed to vaginal surface or mucosa, defined as $5 \mathrm{~mm}$ depth from the applicator surface depending on a boost occurrence. Adjuvant chemotherapy (carboplatin and paclitaxel) was administered to patients with high-grade non-endometroid histology.

The primary endpoints of the present study included disease-free survival (DFS) and disease-specific survival (DSS). Although, all the recurrence sites were noted separately, since we acquired various information with phone calls and we could not confirm certain old hospital data, we chose DFS as the endpoint. Moreover, the majority of patients with this diagnosis were senior with comorbid diseases, therefore we used DSS in order to present the association between variables and survival without interference. The diagnosis date was accepted as the onset point for disease-free and disease-specific survivals. The endpoint for DSS was the last control date for living patients. Patients who died were divided into two groups: as a decease due to the disease or not. Patients who died due to the causes other than endometrial carcinoma were excluded in the DSS analysis. The endpoint for DFS was the first event date for recurrence and distant metastasis, the last control date for patients who have not relapsed.

Adult patients with FIGO stages I-II endometrial cancer who were curatively treated with all accessible information were included in the study. Patients who received palliative RT, had missing files, and follow-up information were excluded from the study.

\section{Statistical analysis}

Statistical analyses were conducted using IBM SPSS Statistics version 20 (SPSS Inc., Chicago, IL, USA), and non-parametric tests were used to assess variables. The categorical demographic characteristics of enrolled patients were calculated with $\chi^{2}$ and Fisher's exact tests. Spearman's rank correlation test was utilized for univariate correlation analysis. Mann-Whitney $U$ test was conducted for two groups of independent statistical analysis and Kruskal-Wallis test was performed for 3 or more independent group analyzes. After Bonferroni correction, the significance was evaluated by post-hoc analysis. The variables studied included the patient age, stage, degree of myometrial invasion, biggest diameter of tumor, tumor grade, lymphovascular invasion, lower uterine segment invasion, and adjuvant treatment. Kaplan-Meier test estimated the survival and with log-rank test were used for univariate analysis. Multivariate Cox regression analysis was performed to determine the effect of variables on survival. The endpoints included progression free survival (PFS) and DSS. All the study outcomes were measured from the date of surgery to address a potential lead time bias. $P$-values of less than 0.05 were considered statistically significant, and all statistical tests performed were 2-sided.

\section{Results}

The data of 311 patients from 2 institutions, with FIGO stage I-II endometrial cancer who underwent curative treatment were retrospectively evaluated. The median age was 60 (range, 33-85) years. All of the patients had surgery, $74(24 \%)$ received no further treatment, 4 (1\%) obtained chemotherapy only, 234 (75\%) received $\mathrm{RT}$, and $24(7 \%)$ received both treatments. The pathology of $277(89.4 \%)$ patients were endometrioid adenocarcino$\mathrm{ma}$, the rest were non-endometrioid type. According to the FIGO staging system, 148 (47.9\%), 108 (34.9\%), and $57(17.2 \%)$ of the patients were stage $\mathrm{Ia}, \mathrm{Ib}$, and II, respectively. Radiotherapy was applied to $106(45.8 \%)$ of the patients as only VBT, and $128(54.2 \%)$ of the patients as both EBRT and VBT. Patients and diseases characteristics are summarized in Table 1.

The median follow-up time was 102 (range, 3-205) months. During this period, 68 (21.9\%) patients died, among whom $41(62.8 \%)$ died due to disease $(n=28)$ or treatment-related $(n=13)$ causes, $24(33.8 \%)$ due to other reasons, and $3(4.4 \%)$ due to unknown causes. Three patients had second primary, with two having breast carcinoma and one bladder carcinoma. During the follow-up time, $26(8.3 \%)$ patients experienced a recurrence, with $15(6.4 \%)$ patients receiving RT and $11(14.2 \%)$ no additional RT.

\section{Results of disease-specific survival}

The median DSS was 101 (range, 1-205) months. The 2-year, 5-year, and 10-year DSS were $93.6 \%, 86.8 \%$, and $82.2 \%$, respectively.

Among the aforementioned variables, the age, LVSI, grade, stage, and adjuvant RT were found to have significant relationship with DSS. The age below 65 years, LVSI, grade 3, stage II disease, and no adjuvant RT were the negative prognostic factors of DSS (Figure 1). Multi- 
Table 1. Patients and disease characteristics

\begin{tabular}{|c|c|}
\hline Characteristics & $n(\%)$ \\
\hline \multicolumn{2}{|l|}{ Age (years) } \\
\hline$<65$ & $208(66.9)$ \\
\hline$\geq 65$ & $103(32.1)$ \\
\hline \multicolumn{2}{|l|}{ Surgery } \\
\hline $\mathrm{TAH}+\mathrm{BSO}+\mathrm{BPPLND}$ & $181(58)$ \\
\hline $\mathrm{TAH}+\mathrm{BSO}$ & $130(42)$ \\
\hline \multicolumn{2}{|l|}{ Pathology } \\
\hline Endometrioid adenocarcinoma & $277(89.4)$ \\
\hline Non-endometrioid adenocarcinoma & $34(10.6)$ \\
\hline \multicolumn{2}{|l|}{ Tumor diameter $(\mathrm{cm})$} \\
\hline$<2$ & $26(8.4)$ \\
\hline $2-4$ & $78(25.1)$ \\
\hline $4.1-6$ & $38(12.2)$ \\
\hline$>6$ & $19(6.1)$ \\
\hline Unknown & $150(48.2)$ \\
\hline \multicolumn{2}{|l|}{ Myometrial invasion } \\
\hline$<0.5$ & $163(52.4)$ \\
\hline$>0.5$ & $148(47.6)$ \\
\hline \multicolumn{2}{|l|}{ Cervical glandular invasion } \\
\hline Yes & $67(21.5)$ \\
\hline No & $244(78.5)$ \\
\hline \multicolumn{2}{|l|}{ Cervical stromal invasion } \\
\hline Yes & $55(17.7)$ \\
\hline No & $256(82.3)$ \\
\hline \multicolumn{2}{|l|}{ Stage (FIGO) } \\
\hline la & $148(47.6)$ \\
\hline $\mathrm{lb}$ & $108(34.9)$ \\
\hline$\|$ & $57(17.2)$ \\
\hline \multicolumn{2}{|l|}{ LVSI } \\
\hline Yes & $172(55.2)$ \\
\hline No & $139(44.8)$ \\
\hline \multicolumn{2}{|l|}{ Grade } \\
\hline 1 & $128(40.8)$ \\
\hline 2 & $131(41.8)$ \\
\hline 3 & $52(17.4)$ \\
\hline \multicolumn{2}{|l|}{ Chemotherapy } \\
\hline Yes & $28(9)$ \\
\hline No & $283(91)$ \\
\hline \multicolumn{2}{|l|}{ Radiotherapy } \\
\hline Yes & $234(75.2)$ \\
\hline No & $77(24.8)$ \\
\hline
\end{tabular}

$T A H+B S O+B P P L N D-$ total abdominal hysterectomy + bilateral salphingooopherectomy + bilateral pelvic paraaortic lymph node dissection, FIGO International Federation of Gynecology and Obstetrics, LVSI - lymphovascular space invasion variate analysis also confirmed the importance of these variables (Table 2).

No significant difference in DSS was noted between the patients receiving either EBRT or VBT (median DSS, 100 (range, 4-142) months vs. 102 (range, 10-205) months).

\section{Results of disease-free survival}

The median DFS was 99 (range, 1-205) months. The 2-year, 5-year, and 10-year DFS were $86 \%, 76 \%$, and $74.3 \%$, respectively.

Disease-free survival was not significantly influenced by cervical stromal involvement $(p=0.19)$, pathological subtype $(p=0.34)$, the use of chemotherapy $(p=0.16)$, tumor size $(p=0.61)$, advanced age $(p=0.43)$, grade $(p=0.14)$, and stage $(p=0.34)$.

A significant correlation was found between lower uterine invasion and DFS $(p=0.015$, hazard ratio $[\mathrm{HR}]=$ 0.19, CI: 0.04-0.85) (Figure 2). The 5-year and 10-year DFS were $73.7 \%$ and $73.5 \%$ vs. $94.2 \%$ and $93.7 \%$ in patients with lower uterine invasion vs. no invasion, respectively.

Similarly, the correlation between adjuvant RT and DFS was significant ( $p=0.017, \mathrm{HR}=4.2, \mathrm{CI}$ : 2.0-8.8) (Figure 2). The 5 -year and 10 -year DFS were $95 \%$ and $94 \%$ vs. $77.3 \%$ and $76 \%$ in patients with RT vs. without RT, respectively. In Table 3, the values of median (range) DFS were indicated according to the factors, which significantly affected DFS.

\section{Discussion}

In this study, we presented the 10-year survival results of 311 patients with early-stage endometrial cancer from two centers, a rather heterogeneous group with different approaches. All patients underwent surgery, 74 (24\%) received no further treatment, $4(1 \%)$ obtained chemotherapy only, $234(75 \%)$ received $\mathrm{RT}$, and $24(7 \%)$ received both treatments. The 5-year and 10-year DFS were $76 \%$ and $74.3 \%$, respectively. In multivariate analysis, lower uterine segment invasion positivity and no adjuvant RT were determined as independent unfavorable prognostic factors for DFS. The 5-year and 10-year DSS were $86.8 \%$ and $82.2 \%$, respectively. For DSS, the high-grade, LVSI positivity, stage II, $\geq 65$ age, and no adjuvant RT were found to be the independent unfavorable prognostic markers. As a result of the present study, adjuvant RT in early-stage endometrial cancer is a significant independent prognostic factor for both disease-free and disease-specific survival.

Adjuvant RT has a well-defined role in early-stage endometrial carcinoma; post-operative EBRT reduces isolated relapses from $14-12 \%$ to $4-3 \%$, absolute benefit of nearly $10 \%$ in stage I patients [4,5]. In our cohort, comprised of stage I and II, $6.4 \%$ of patients who received RT experienced recurrence, a quite comparable result. Patients with stage I, grade 1 , or grade 2 with $<50 \%$ myometrial invasion and endometrioid histology without other risk factors, are generally considered low-risk, and expected recurrence rate is $<5 \%$ [9]. Unexpectedly, the rate of recurrence rate was quite high in our patients without adjuvant RT. We presume that some of the patients with risk factors already necessitating adjuvant treatment did 


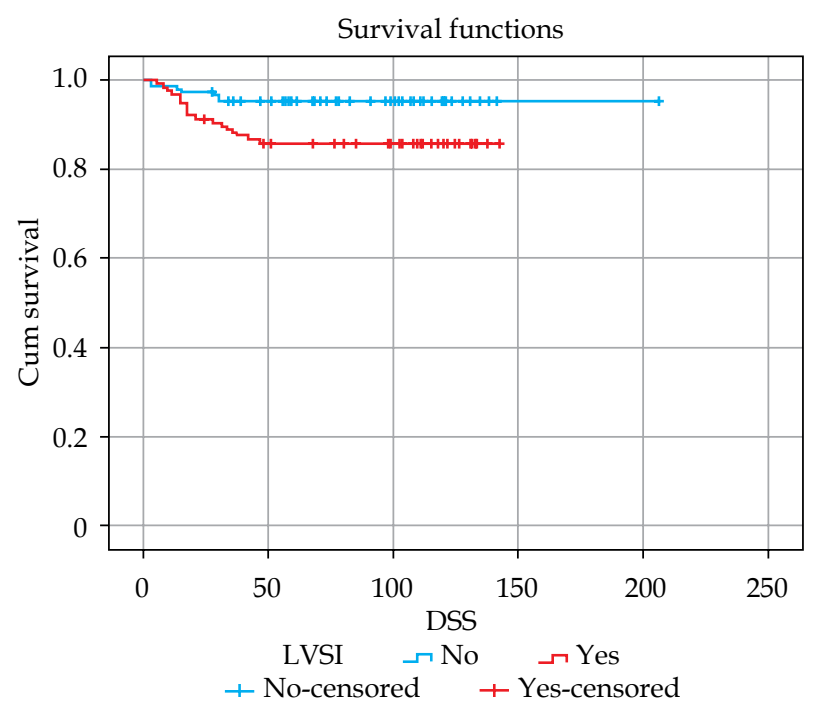

Survival functions

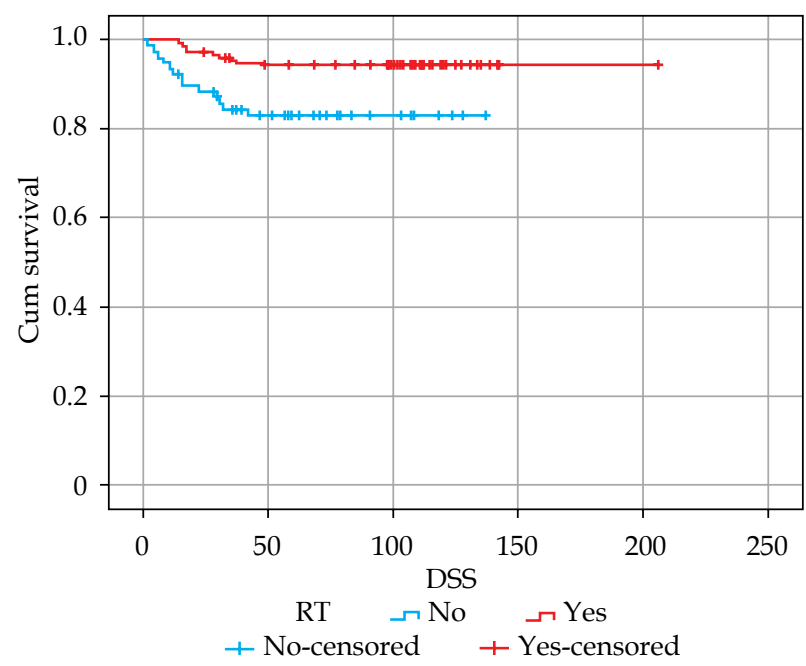

Survival functions

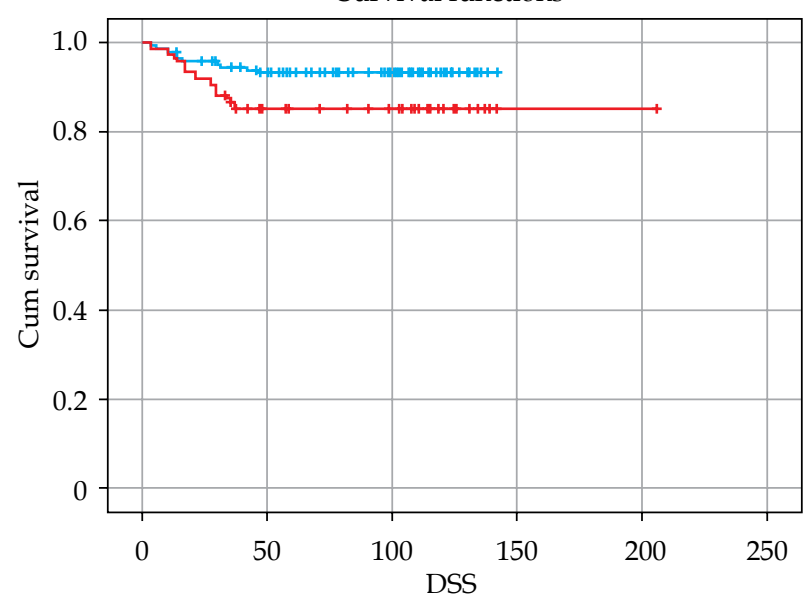

Age $\rightarrow 65$ under $\rightarrow 65$ andover +65 under-censored +65 andover-censored

not receive RT, either because they were not offered or the pathology underscored grade or degree of myometrial invasion; this emphasize the importance of central pathology review in randomized trials.
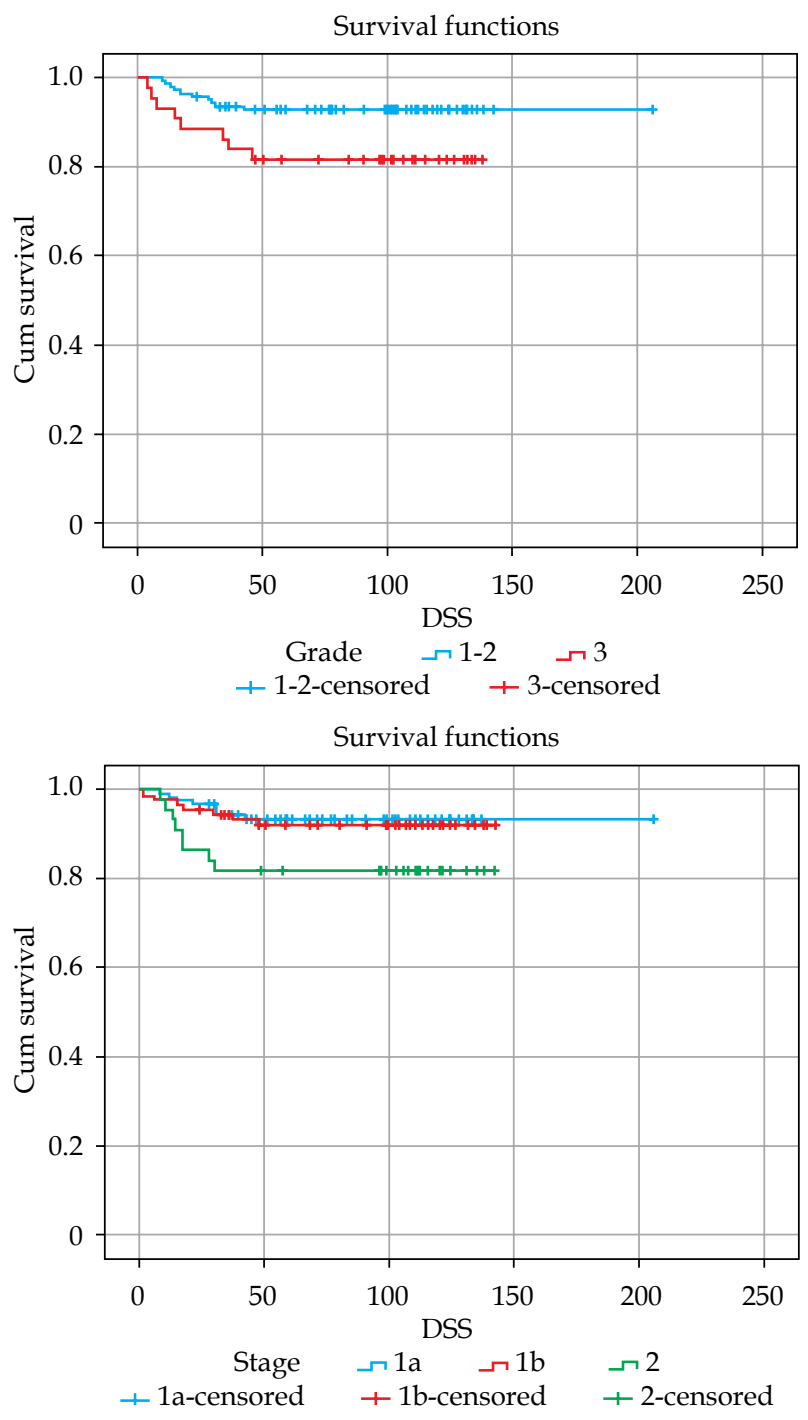

Fig. 1. Survival curves of the factors that significantly improve disease-specific survival (DSS)

The advantage of chemoradiotherapy over RT alone was not demonstrated in early-stage endometrial cancer [10]. In the recently published PORTEC-3 multicenter phase 3 study, the benefits of adjuvant chemotherapy 
Table 2. Detailed analysis of disease-specific survival (DSS) according to the factors significantly affecting DSS

\begin{tabular}{|c|c|c|}
\hline $\begin{array}{l}\text { Factors that significantly } \\
\text { improve DSS }\end{array}$ & $\begin{array}{c}\text { DSS, } \\
\text { median (range), } \\
\text { months }\end{array}$ & $P$-value \\
\hline LVSI & & 0.010 \\
\hline Positive & $91(2-142)$ & \\
\hline Negative & $107(1-205)$ & \\
\hline Grade & & 0.014 \\
\hline $1-2$ & $101(1-205)$ & \\
\hline 3 & 99 (3-137) & \\
\hline Stage & & 0.048 \\
\hline la & $102(3-205)$ & \\
\hline $\mathrm{lb}$ & $102(7-142)$ & \\
\hline II & $100(1-141)$ & \\
\hline Age (years) & & 0.038 \\
\hline$<65$ & $100(1-142)$ & \\
\hline$\geq 65$ & $87(2-205)$ & \\
\hline RT & & 0.002 \\
\hline Yes & $107(2-205)$ & \\
\hline No & $51(1-136)$ & \\
\hline
\end{tabular}

LVSI-lymphovascular space invasion, $R T$-radiotherapy, DSS - disease-specific survival

and pelvic RT in high-risk endometrial cancer patients were examined. Patients were randomized to pelvic RT and chemotherapy (2 cycles of cisplatin concurrent with RT, and 4 cycles of carboplatin and paclitaxel following RT) and pelvic RT only (48.6 Gy) arms. In stage I-II patients, the advantage of survival was not achieved with adjuvant chemotherapy. In a subgroup analysis, adjuvant chemotherapy increased failure-free survival (FFS) only in stage III disease. This study demonstrated that pelvic control was achieved only by RT in early-stage endometrial cancer patients. In a review for evaluating adju-

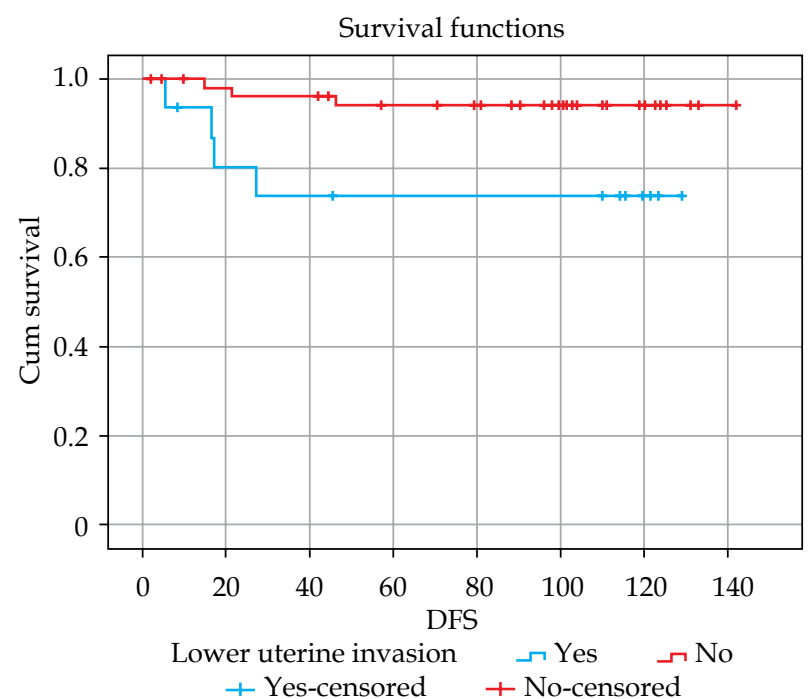

vant chemoradiotherapy versus RT alone in early-stage endometrial cancer patients, the superiority of adjuvant chemoradiotherapy over RT alone was not demonstrated in overall survival (OS) and FFS [11]. In the GOG 249 phase 3 study [12], 601 patients with high-risk earlystage endometrial cancer were randomized to pelvic RT, with 3 cycles of paclitaxel/carboplatin, and VBT arms. The primary endpoint was recurrence-free survival. However, the superiority of VBT plus chemotherapy over pelvic RT could not be revealed. The authors propounded that pelvic RT was the most effective and optimal adjuvant treatment option in early-stage endometrial cancer [12]. In a recent study, chemotherapy was applied, only in $9 \%$ of patients, which did not impact survival parameters.

Lin et al. [13] examined the role of adjuvant RT in 337 stage I endometrial cancer patients from a single center. 5 -year OS and 5-year loco-regional recurrence-free survival (LR-RFS) were $96.3 \%, 97.2 \%$ for the group without $\mathrm{RT}$, and $91.6 \%, 97.1 \%$ for the group with RT, respectively $(p=0.06$ for OS and $p=0.956$ for LR-RFS). In a multivariate analysis, $>60$ years of age and $>50 \%$ myometrial invasion were significant prognostic factors for OS. They revealed pre-operative CA-125 level, > 60 years of age, LVSI, and adjuvant RT as independent prognostic factors for LR-RFS. In the conclusion of the study, adjuvant RT was an independent predictor for LR-RFS. However, the authors failed to demonstrate its effect on OS. Similarly, in our study, the advanced age, absence of adjuvant RT, and LVSI positivity were found to be independent prognostic factors for DSS. In two other similar retrospective studies, the presence of LVSI has been denoted to be associated with vaginal relapse and poor OS $[14,15]$.

Yilmaz et al. [16] investigated the impact of adjuvant RT on survival in patients with early-stage high-grade endometrial cancer. Adjuvant RT was administrated to $57 \%$ of patients as pelvic RT (average dose of $46 \mathrm{~Gy}$ ) and the rest as VBT (average dose of 21.5 Gy). There was no significant relationship between adjuvant RT and overall and DFS. Elevated serum CA-125 level and histological

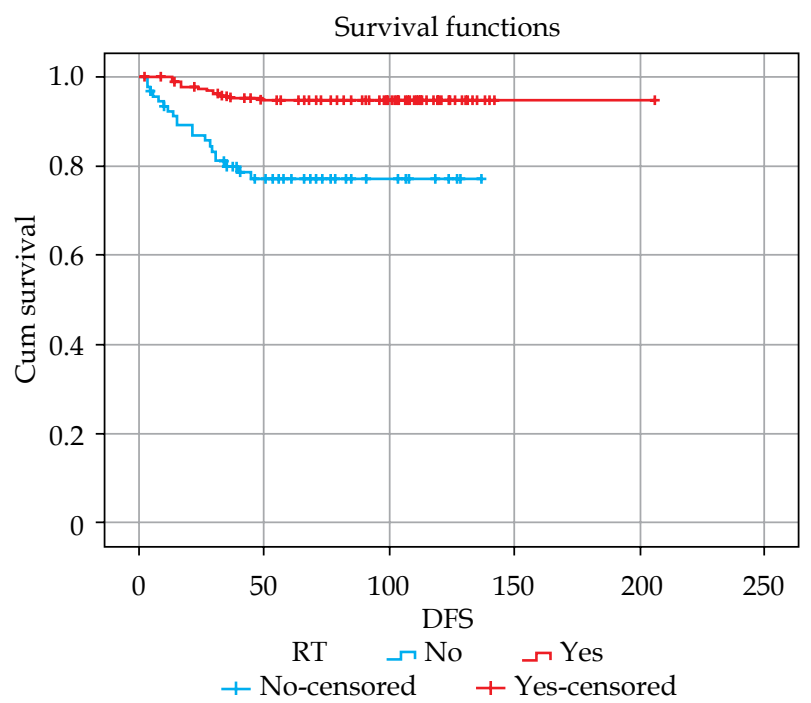

Fig. 2. Survival curves of the factors that significantly improve disease-free survival (DFS) 
Table 3. Detailed analysis of disease-free survival (DFS) according to the factors significantly affecting DFS

\begin{tabular}{|c|c|c|}
\hline $\begin{array}{l}\text { Factors that significantly } \\
\text { improve DFS }\end{array}$ & $\begin{array}{c}\text { DFS, } \\
\text { median (range), } \\
\text { months }\end{array}$ & $P$-value \\
\hline Low uterine invasion & & 0.015 \\
\hline Presence & $101(6-128)$ & \\
\hline Absence & $112(4-142)$ & \\
\hline RT & & 0.017 \\
\hline Yes & $106(6-205)$ & \\
\hline No & $50.8(4-136)$ & \\
\hline
\end{tabular}

$R T$ - radiotherapy, DFS - disease-free survival

grade were independent predictors for DFS. In contrast, in the present study, the adjuvant RT was an independent prognostic factor for both disease-specific and disease-free survival. We indicated that the absence of adjuvant RT and lower uterine segment invasion were negative prognostic factors for DFS.

Due to the concerns about potential toxicity of EBRT, the PORTEC-2 trial was designed to illustrate if VBT would be sufficient instead of EBRT as an adjuvant RT in early-stage endometrial cancer [6]. This trial was non-inferior study comparing EBRT and VBT alone. Vaginal recurrence was $0.9 \%$ and $2 \%$ in the VBT alone and EBRT arms, respectively, and this difference was not statistically significant. Furthermore, there was no statistical difference between the two arms at 5-year OS rates $(82 \%$ vs. $86 \%$ ). Vaginal brachytherapy has been indicated to cause less morbidity than EBRT, while providing similar vaginal tumor control. Kim et al. [17] reported the treatment results of post-operative VBT and/or EBRT in patients with stage I endometrial cancer. The 5-year DFS was $88 \%$ in the EBRT group and $96 \%$ in the VBT group. Similarly, the 5-year OS was $94 \%$ in the EBRT group and $96 \%$ in the VBT group. The results were not significant due to low number of patients and events. However, they argued that VBT may be preferred in selected patients due to lower than EBRT toxicity and similar treatment outcomes [17]. In our series, we also demonstrated no survival difference between patients receiving EBRT and VBT.

In the present study, the main goal was to report long-term survival results of patients with early-stage endometrial cancer. Data presented the results of 15-year follow-up, and these results are the conclusion of the actual treatment executed without any randomization as in phase 3 studies, which reflects the real-world data, and makes the current study valuable. Moreover, we reported 10 years of survival in this study, whereas many studies in the literature reported 5 years of survival $[4,5,10]$. We submitted the results of DFS similar to FFS: the 5-year DFS was $76 \%$ and the 10 -year DFS was $74 \%$. In the literature, 5-year DFS rates for patients with early-stage endometrial cancer ranged from $75 \%$ to $88 \%[16,17,18]$.

This study has some limitations. It was retrospective in nature involving a heterogeneous group of patients. Failure to separate the treatments according to the risk groups restricts the study. Moreover, differentiating the site of recurrence could help to understand the results better. This study evaluated data of patients from a period of 15 years, and potential variation/evolution in standards of management can be a disadvantage while interpreting results.

\section{Conclusions}

At 15 years of follow-up, the adjuvant RT has been shown to be the significant independent prognostic factor for both DSS and DFS. Radiotherapy should remain the essential part of the treatment in the management of early-stage endometrial cancer.

The abstract of current study was presented as an oral presentation in the Second Black Sea Gynecology and Obstetrics Congress.

\section{Disclosure}

The authors report no conflict of interest.

\section{References}

1. Bray F, Ferlay J, Soerjomataram I et al. Global cancer statistics 2018: GLOBOCAN estimates of incidence and mortality worldwide for 36 cancers in 185 countries. CA Cancer J Clin 2018; 68: 394-424.

2. Ferguson SE, Panzarella T, Lau S et al. Prospective cohort study comparing quality of life and sexual health outcomes between women undergoing robotic, laparoscopic and open surgery for endometrial cancer. Gynecol Oncol 2018; 149: 476-483.

3. Pecorelli S. Revised FIGO staging for carcinoma of the vulva, cervix, and endometrium. Int J Gynaecol Obstet 2009; 105: 103-104.

4. Creutzberg CL, van Putten WL, Koper PC et al. Surgery and postoperative radiotherapy versus surgery alone for patients with stage I endometrial carcinoma: multicentre randomised trial. PORTEC Study Group. Post Operative Radiation Therapy in Endometrial Carcinoma. Lancet 2000; 355: 1404-1411.

5. Keys HM, Roberts JA, Brunetto VL et al. A phase III trial of surgery with or without adjunctive external pelvic radiation therapy in intermediate risk endometrial adenocarcinoma: a Gynecologic Oncology Group study. Gynecol Oncol 2004; 92: 744-751.

6. Nout RA, Smit VT, Putter H et al. Vaginal brachytherapy versus pelvic external beam radiotherapy for patients with endometrial cancer of high-intermediate risk (PORTEC-2): An open-label, non-inferiority, randomised trial. Lancet 2010; 375: 816-823.

7. Johnson N, Cornes P. Survival and recurrent disease after postoperative radiotherapy for early endometrial cancer: systematic review and meta-analysis. BJOG 2007; 114: 1313-1320.

8. Onsrud M, Cvancarova M, Hellebust TP et al. Long-term outcomes after pelvic radiation for early-stage endometrial cancer. J Clin Oncol 2013; 31: 3951-3956.

9. Sorbe B, Nordström B, Mäenpää J et al. Intravaginal brachytherapy in FIGO stage I low-risk endometrial cancer: A controlled randomized study. Int J Gynecol Cancer 2009; 19: 873-878.

10. de Boer SM, Powell ME, Mileshkin L et al. Adjuvant chemoradiotherapy versus radiotherapy alone for women with high-risk endometrial cancer (PORTEC-3): final results of an international, open-label, multicentre, randomised, phase 3 trial. Lancet Oncol 2018; 19: 295-309. 
11. Jingjing H, Rui J, Hui P. Adjuvant chemoradiotherapy vs. radiotherapy alone in early-stage high-risk endometrial cancer: a systematic review and meta-analysis. Eur Rev Med Pharmacol Sci 2019; 23: 833-840.

12. Randall ME, Filiaci V, McMeekin DS et al. Phase III Trial: adjuvant pelvic radiation therapy versus vaginal brachytherapy plus paclitaxel/carboplatin in high-intermediate and high-risk early stage endometrial cancer. J Clin Oncol 2019; 37: 1810-1818.

13. Lin YJ, Hu YW, Twu NF et al. The role of adjuvant radiotherapy in stage I endometrial cancer: A single-institution outcome. Taiwan J Obstet Gynecol 2019; 58: 604-609.

14. Cusano E, Myers V, Samant R et al. Prognostic significance of lymphovascular space invasion in the absence of lymph node metastases in early-stage endometrial cancer. Int J Gynecol Cancer 2018; 28: 890-894.

15. Mariani A, Dowdy SC, Keeney GL et al. Predictors of vaginal relapse in stage I endometrial cancer. Gynecol Oncol 2005; 97: 820-827.

16. Yilmaz E, Gurocak S, Melekoglu R et al. The effect of prognostic factors and adjuvant radiotherapy on survival in patients with high-grade early-stage endometrial cancer: a retrospective clinical study. Med Sci Monit 2019; 25: 2811-2818.

17. Kim J, Lee KJ, Park KR et al. Treatment outcomes after adjuvant radiotherapy following surgery for patients with stage I endometrial cancer. Radiat Oncol J 2016; 34: 265-272.

18. Amant F, Moerman P, Neven P et al. Endometrial cancer. Lancet 2005; 366: 491-505. 